\title{
Development of a potential vortex-hazard index to predict cruise-level wake turbulence encounters
}

\author{
J SARAVANAKUMAR, C ARSHAD SHAMEEM and T N VENKATESH* \\ CTFD Division, CSIR-National Aerospace Laboratories, PB 1779, Bengaluru 560017, India \\ e-mail: saravanakumarjayaraj@yahoo.com; arshad@nal.res.in; tnv@nal.res.in
}

MS received 14 April 2018; revised 3 December 2018; accepted 17 December 2018; published online 21 March 2019

\begin{abstract}
A pair of counter-rotating trailing vortices formed as a consequence of lift generation poses a potential threat to the encountering aircraft. In recent years, incidents/accidents involving wake turbulence confrontation at cruise altitude are increasing due to increase in the number of super-heavy category aircraft. The vertical separation distance between two airplanes guided by the Federal Aviation Administration (FAA) could be inadequate in certain cases. In this article, a new approach has been proposed to predict the probable area of the persistence of trailing vortices produced by large aircraft. It includes the development of an index with multiple atmospheric effects on the vortices. Also, the impact of the wake from a distinct airplane is incorporated. The performance assessment of the index in predicting the cruise-level wake vortex encounters has been made using various scenarios that happened in the recent past. Weather Research and Forecasting (WRF) model is employed to simulate the atmospheric flow conditions of chosen situations. We found that the method successfully predicts all the cases considered in this study.
\end{abstract}

Keywords. Aviation weather hazards; aircraft wake vortices; potential vortex-hazard index; cruise-level encounters; wake turbulence; WRF.

\section{Introduction}

Impacts of prevailing meteorological conditions on civil aviation are well known and weather considerably affects the safety of both passengers and airplane. Besides flight safety, airline operations depend substantially on weather phenomena for flight planning, maintenance schedules, departure and arrival of the airplanes. In recent times, possible effects of growing air traffic and global warming have dramatically raised the number of incidents/accidents involving atmospheric phenomena. Aviation weather hazards include convective turbulence (activities near the clouds), clear-air turbulence (CAT), wind shear and aerodynamic (wake) turbulence. Almost $7 \%$ of the aviation weather hazard events that occurred in the US from 1987 till 2011 were due to wake turbulence [1].

A potential safety issue of wake vortex encounters at cruise levels is anticipated [2,3] because of increasing air traffic, utilization of large transport aircraft like Airbus A380 and Boeing 747 by the airliners and the reduced vertical separation between two aircraft. A Wake Encounter Avoidance and Advisory system (WEAA) has been developed at German Aerospace Center and validated using data collected from flight tests $[4,5]$. At cruise altitude, though on-board systems and forecasting techniques are

*For correspondence used to identify the presence of some of the weather hazards, currently there are no built-in systems to show the persistence of the trailing vortices behind the aircraft. This is not a weather phenomenon, but is greatly influenced by the local atmospheric conditions.

It is well known that an aircraft generates wake turbulence during its course (take-off, cruise and landing) as a consequence of the lift generation. The vortex sheet shed from the trailing edge of the wing eventually rolls up into a pair of counter-rotating vortices within a few spans downstream of the wings. These vortices pose a potential threat by imposing rolling moments, altitude loss and even structural damage to the following or encountering aircraft. While vortex interaction has been studied widely for takeoff and landing conditions, recent encounters at cruise level due to the trailing vortices of Airbus A380 and Boeing 747 highlight the need to study this problem: characterization of the persistence and movement of these vortices at cruise levels in the atmosphere.

The behaviour of trailing vortices at cruise levels is quite different from those at take-off and landing conditions since the ground effect is absent. Also the prevailing atmospheric conditions are very different compared with the boundary layer flow near the runway. The initial strength and propagation of the wake vortices primarily depend on specifications (mass, wingspan and speed) of the generating aircraft and at cruise level, the effect of air density plays an 
important role. To ensure the safety of air travel, Federal Aviation Administration (FAA) has advised the minimum standard separation distance (both in lateral and vertical directions) between the two aircraft [6]. Reduced Vertical Separation Minimum (RVSM) implemented by FAA reduced the vertical separation distance between two aircraft from 2000 to 1000 feet $(300 \mathrm{~m})$ at flight levels from 29,000 up to 41,000 feet. Because of the prevailing weather conditions that significantly determine the decay rate and propagation of the vortices in the atmosphere, it has been suggested that the reduced vertical separation could be inadequate for Airbus A380 and Boeing 747. Atmospheric turbulence and stable atmosphere are the conditions that increase the decay rate of wake vortices and are therefore the conducive conditions for vortex encounters [7].

A pair of counter-rotating vortices descend in the airspace by mutual interaction. Also, there are various factors like atmospheric turbulence, stratification, crosswind, crosswind shear and its vertical gradient present in the atmosphere that affect the strength and propagation of the vortices. Atmospheric turbulence enhances the decay rate of the vortices [8], and rapid diffusion of vorticity in the turbulent condition decreases the descent speed of the vortices over the time [9]. In general, a mass of fluid (with oval boundary) carried by the descending vortices along with them will encounter fluid of different density due to stratification of the atmosphere [10]. At cruise conditions, the strongly stable stratification usually restricts the descent rate due to developing buoyancy force and enhances the decay rate of the descending vortices [11]. In this study, the atmospheric stratification effects on descending vortices are not considered explicitly.

Typically, crosswind will advect the descending vortices from the flight path of the generating aircraft. Based on the sign of vertical gradient of crosswind shear, the vortex pair will tilt either in clockwise or counter-clockwise direction $[12,13]$ and vortex pair will no longer descend in a purely vertical direction. Once the vortices get tilted, crosswind shear transports the vortices at different lateral speeds [14] and alters the vortex decay and descent rate of the vortices. In the context of increasing airport capacity, Hallock and Holzapfel [15] gave a brief overview of contemporary research on aircraft wake vortices.

A preliminary study was conducted by us [16], in which we showed that the wake vortex encounters at higher altitudes are predictable. In this article, a potential vortexhazard index is proposed by combining the newly developed two-dimensional wake vortex model and atmospheric conditions from a weather model. The index indicates the possible area of the persistence of trailing vortices generated by large transport aircraft at cruise level. It also incorporates various atmospheric effects on descending vortices and the effect of all the vortices from different aircraft. Atmospheric turbulence as a measure of turbulence kinetic energy is defined, which determines the decay rate of the vortices over time. We have conducted performance assessment tests of the index in predicting whether the wake turbulence encounter will happen or not with several identified test-cases.

Stratification effects, though important, are not included in our model at present. These effects would be significant in regions with strong stable stratification. Buoyancy would reduce the descent velocity and lifetimes of the vortices. Under those conditions, the estimates from our model would be higher. Inclusion of these effects would further enhance the model and is planned as a part of subsequent study.

The structure of this article is as follows. A brief description of the cruise-level wake vortex model proposed for assessing vortex encounters is presented in section 2 . Details of the methodology proposed for predicting the possible area of the persistence of the vortices descending from the cruising aircraft, atmospheric models and types of grids used are discussed in section 3. The performance assessment of the index made with several identified test cases is presented in section 4. Finally, conclusions are drawn in section 5 .

\section{Cruise-level wake vortex model}

A new model for assessing aircraft wake encounters at cruise altitude is developed [17], which includes several atmospheric effects on descending vortices. Decay and propagation of the vortices in a plane perpendicular to the generating aircraft flight path $(y-z$ plane $)$, idealized as a two-dimensional problem, are studied. Decay due to atmospheric turbulence is modelled following Donaldson and Bilanin [9]. Cross-wind effects on the decay of wake vortices are incorporated following works of Delisi and Robins [12], Robins and Delisi [13] and Proctor and Ahmad [14]. The strength and position of the vortices in the $y-z$ plane can be determined using the following ODEs. They are solved numerically by applying a fourth-order Runge-Kutta time integration scheme.

$$
\begin{gathered}
\frac{d \Gamma_{1}}{d t}=-0.41 q \frac{\Gamma_{1}}{r_{12}} \\
\frac{d \Gamma_{2}}{d t}=-0.41 q \frac{\Gamma_{2}}{r_{12}} \\
\frac{d y_{1}}{d t}=\frac{1}{2 \pi}\left[\frac{\Gamma_{2}\left(z_{2}-z_{1}\right)}{\left(y_{2}-y_{1}\right)^{2}+\left(z_{2}-z_{1}\right)^{2}}\right]+\Omega r_{12} \sin \Theta+V_{E} \\
\frac{d y_{2}}{d t}=\frac{1}{2 \pi}\left[\frac{\Gamma_{1}\left(z_{1}-z_{2}\right)}{\left(y_{2}-y_{1}\right)^{2}+\left(z_{2}-z_{1}\right)^{2}}\right]-\Omega r_{12} \sin \Theta+V_{E}
\end{gathered}
$$




$$
\begin{aligned}
& \frac{d z_{1}}{d t}=-\frac{1}{2 \pi}\left[\frac{\Gamma_{2}\left(y_{2}-y_{1}\right)}{\left(y_{2}-y_{1}\right)^{2}+\left(z_{2}-z_{1}\right)^{2}}\right]-\Omega r_{12} \cos \Theta+w_{p} \\
& \frac{d z_{2}}{d t}=-\frac{1}{2 \pi}\left[\frac{\Gamma_{1}\left(y_{1}-y_{2}\right)}{\left(y_{2}-y_{1}\right)^{2}+\left(z_{2}-z_{1}\right)^{2}}\right]+\Omega r_{12} \cos \Theta+w_{p}
\end{aligned}
$$

where

$$
\begin{aligned}
r_{12} & =\frac{1}{2} \sqrt{\left(y_{1}-y_{2}\right)^{2}+\left(z_{1}-z_{2}\right)^{2}} \\
\Omega & =\int \frac{1.42 w}{\pi} \frac{d^{2} V_{E}}{d z^{2}} d t \\
\Theta & =\tan ^{-1}\left(\frac{z_{2}-z_{1}}{y_{2}-y_{1}}\right)
\end{aligned}
$$

and the subscripts 1 and 2 refer to port and starboard tip vortices, respectively.

Based on the results from the model, an approximate elliptical area influenced by the vortices during its propagation in the atmosphere is estimated. The elliptical boundary is calculated with a threshold value where the magnitude of the induced velocity is $1 \mathrm{~m} / \mathrm{s}$. This elliptical region could be the potential threat to the encountering aircraft. Empirical relations for calculating the length of semi-major and semi-minor axes of the elliptical boundary and the maximum time for which the vortices can persist in the atmosphere enclosing the air masses are proposed, which are used for rapid computation in the prediction process (details are given in the next section).

\section{Potential vortex-hazard index}

Coupling the cruise-level wake vortex model and atmospheric conditions from the weather model, a potential vortex-hazard index $[18,19]$ is defined that represents the possible area of the persistence of the trailing vortices produced by the generating aircraft at cruise level. With the location of the encountering aircraft, it can predict whether the wake turbulence encounter will occur or not. It incorporates the effect of all the vortices from different generating aircraft if the air-traffic density is high.

\subsection{Grids used in the study}

The coupling of the atmospheric model and vortex model for computing potential vortex-hazard index has been made using two different types of grids.

3.1a Met-grid: The atmospheric weather condition simulations are performed using met-grid. The grid resolution depends on the type of atmospheric model (Global
Circulation Model or Regional Model) used. Either analyses or forecast data can also be used. For the current study, Weather Research and Forecasting (WRF) model [20], a public domain, limited area model of NCAR is used to simulate the local weather conditions. It is a non-hydrostatic primitive equation model with multiple options for solving various sub-grid scale physical processes and many run-time options.

An Advanced Research WRF (ARW) dynamic solver with Arakawa C-grid staggering for the horizontal grids is used to solve a fully compressible system of equations. Initial and lateral boundary conditions are from NCEP-FNL datasets. Four domains with a horizontal resolution of $27 \times$ $9 \times 3 \times 1 \mathrm{~km}$ and vertical resolution of 50 model levels are used. The results presented in this paper are from $27-\mathrm{km}$ horizontal resolution computational domain. Table 1 lists summary of the physics and dynamics options used in the WRF model for simulating the weather conditions of the identified test cases at a resolution of $27 \mathrm{~km}$.

$3.1 \mathrm{~b}$ Flight grid: The persistence of the trailing vortices in the atmosphere is calculated using a flight grid. In this study, a flight grid with the horizontal resolution of $25 \mathrm{~km}$ and $25 \mathrm{~m}$ vertical resolution is used. A schematic representation of the trailing vortices from a distance along the flight path of the generating aircraft at different time $\tau$ in the flight grid $(x-z$ plane $)$ is presented in figure 1.

\subsection{Prediction method}

The method for prediction begins with calculating the state of the vortices descending from the generating aircraft along its path in the flight grid. The initial state of the

Table 1. Summary of the physics and dynamics options used in the WRF simulations at a resolution of $27 \mathrm{~km}$.

\begin{tabular}{lc}
\hline Physics & \\
Micro-physics & WSM 3-class simple ice scheme \\
Longwave radiation & RRTM scheme \\
Shortwave radiation & Dudhia scheme \\
Surface layer & Monin-Obukhov scheme \\
Land surface & Thermal diffusion scheme \\
Boundary layer & YSU scheme \\
Cumulus scheme & Kai-Fritsch (new eta) scheme \\
Dynamics & Runge-Kutta 3rd order \\
Time-integration & \\
scheme & w-Rayleigh damping \\
Upper level damping & with damping \\
Vertical velocity & \\
damping & 5 \\
Horizontal momentum \\
advection order \\
Vertical momentum \\
advection order
\end{tabular}

Default schemes and values in WRF are used for other options. 


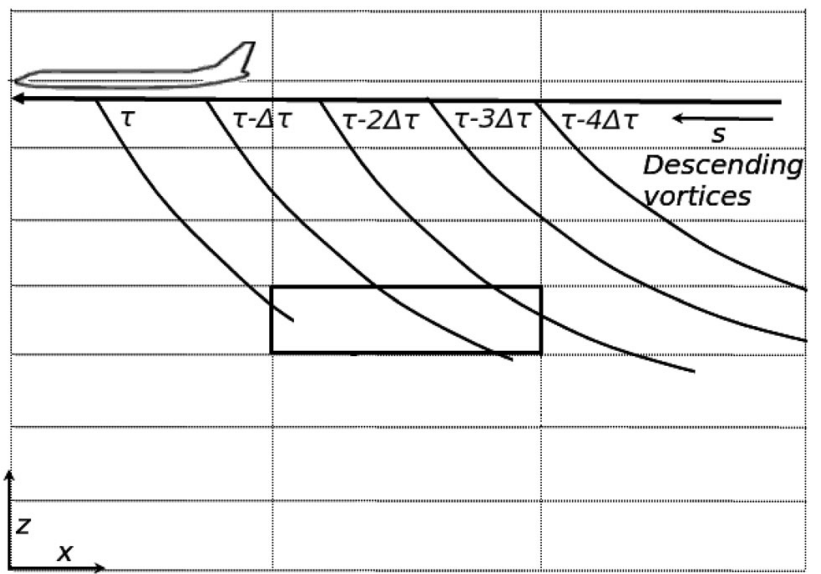

Figure 1. Schematic representation of the descending vortices in the flight grid at different times $\tau$. The dark rectangular box indicates the target flight grid.

vortices primarily depends on the specifications of the generating aircraft and air density at that altitude, given by

$$
\begin{gathered}
\Gamma_{0}=\frac{m g}{\rho_{c} V_{c} R_{12}}, \\
w_{0}=\frac{\Gamma_{0}}{4 \pi r_{12}} .
\end{gathered}
$$

From the earlier studies with the cruise-level wake vortex model, simplified relations for the strength and motion of the descending vortices in the airspace have been derived. The state of the vortices at any $\tau$ is given by

$$
\begin{aligned}
& \Gamma(\tau)=\Gamma_{0} e^{\left(-0.41 q \tau / r_{12}\right)}, \\
& w(\tau)=w_{0} e^{\left(-0.41 q \tau / r_{12}\right)} .
\end{aligned}
$$

Here, atmospheric turbulence is considered to be the main source for the decay of the vortices. Based on multiple experiments with the two cases described in sections 4.1 and 4.2 , called the training set, atmospheric turbulence as a measure of turbulence kinetic energy is defined as

$$
q=\left\{\begin{array}{cc}
k_{s} l_{s} \frac{d \vec{V}}{d z} & \text { if } U<20 \mathrm{~m} / \mathrm{s} \\
\sqrt{2 e} & \text { if } U \geq 20 \mathrm{~m} / \mathrm{s}
\end{array}\right.
$$

where

$$
\begin{aligned}
e & =\frac{3}{2}(U I)^{2} \\
U & =\sqrt{u_{p}^{2}+v_{p}^{2}+w_{p}^{2}}
\end{aligned}
$$

and $\frac{d \vec{V}}{d z}$ is the vertical wind shear. The length scale $l_{S}=\Delta z$ depends on the vertical levels of the met-grid, and turbulence intensity $I=2 \%$ is used.
After performing several analyses, with two critical cases chosen as the training set, the empirical value of $k_{s}=0.38$ was chosen. This was then used for the rest of the test cases.

The location of the vortices at any $\tau$ is given by

$$
\begin{gathered}
x_{c}(\tau)=V_{c} \tau \cos \theta+V_{E} \tau \sin \theta, \\
y_{c}(\tau)=-V_{c} \tau \sin \theta+V_{E} \tau \cos \theta, \\
z_{c}(\tau)=\int_{0}^{\tau} w(\tau) d \tau+w_{p} \tau,
\end{gathered}
$$

where

$$
\begin{aligned}
\theta & =\tan ^{-1}\left(\frac{\varphi_{t}-\varphi_{t-\Delta t}}{\lambda_{t}-\lambda_{t-\Delta t}}\right), \\
V_{E} & =v_{p} \cos \theta-u_{p} \sin \theta .
\end{aligned}
$$

If ground speed is used for cruise velocity $V_{c}$, then it should be corrected with the tailwind/headwind:

$$
V_{T}=u_{p} \cos \theta+v_{p} \sin \theta .
$$

All these calculations are performed till the maximum time for which the vortices can persist in the atmosphere [17], enclosing air masses:

$$
\tau_{\max }=5.5 \frac{r_{12}}{q} .
$$

Here, $x_{c}, y_{c}$ and $z_{c}$ are the centre location of the approximate elliptical area affected by the descending vortices in space at time $\tau$. To capture the effect of all the vortices descending from the generating aircraft at different $\tau$, we have considered the lower of the two values, $4 \tau_{\max }$ and $10 \mathrm{~min}$, as the maximum period for the computation. The cut-off of $10 \mathrm{~min}$ is to account for certain cases where the $q$ is very low, leading to unrealistically high estimates of vortex lifetimes. Parametric studies conducted showed that there is no significant change in the results for cut-off times above $5 \mathrm{~min}$. With the known location (longitude, latitude and height) of the generating aircraft at time $t$ as the starting point $\left(x_{c}, y_{c}, z_{c}\right)$, the calculations are performed from 0 to a maximum period. The estimated position of the descending vortices, including its strength at different $\tau$, is oriented in the flight grid along the flight path of the generating aircraft.

The influence of the vortices at each point in the flight grid, which represents the probable area of the persistence of the vortices at given $t$, defines a potential vortex-hazard index as follows:

$$
\psi(i, j, k, t)=\sum_{\beta} \frac{\Gamma_{\beta}(i, j, k, t)}{\Gamma_{c}}
$$

where $i, j$ and $k$ are indices along $x, y$ and $z$ directions in the flight grid, respectively, and $\beta$ is the position of the vortices contributing to the index at given $t$ on a flight grid. The 
circulation $\Gamma_{\beta}(i, j, k, t)$ is normalized with a constant circulation value $\Gamma_{c}$. This index includes the addition of the vortices from distinct aircraft if more than one generating aircraft is considered at $t$ due to high air-traffic density.

\section{Performance assessment and results}

The usefulness of the potential vortex-hazard index in predicting whether the wake turbulence encounter at cruise level will happen or not is assessed using different circumstances (test cases) that happened in the recent past. The selection of test cases plays a significant part because of the availability of the required data.

For many of the reported cases, only partial information is available in the public domain, which is not sufficient to assess the performance of the vortex-hazard index. Hence, we have chosen a few recent-occurrence wake turbulence encounter test cases for which the required data are available in the public domain.

Also, we examined the effectiveness of the index in predicting the no-encounter circumstance where the Airbus A380 and Boeing 747 had crossed the other aircraft with 1000 feet vertical separation, but there are no records available in the public domain. The test cases for no-encounter state are collected by continuously observing the air traffic from the websites www.flightradar24.com and www.flightaware.com/live/.

Details of all the identified test cases are discussed in the following sections. Of these, the first two form the training set and the remaining case, the test set.

\section{1 $7^{\text {th }}$ January 2017 wake vortex encounter case}

On $7^{\text {th }}$ January 2017, Canadair CL604 Challenger, a private jet encountered the wake turbulence of Airbus A380 while performing flight number MHV-604 from Male to Abu Dhabi. The Airbus A380 was flying in the opposite direction of CL604, performing flight number EK-412 from Dubai to Sydney with 1000 feet vertical separation. The approximate flight paths followed by the two aircraft are shown in figure 2. From the interim report [21], it was found that the accident took place around 08:40Z approximately 5-10 NM (nautical miles) away DONSA waypoint. A few minutes before the incident, the flight crew of CL604 noticed Airbus A380 flying above them at 35,000 feet slightly offset to their left. The business jet CL604 lost about 9000 feet before the pilot regained control, and it was diverted to Muscat due to severe injuries to the passengers and crew members. At the time of the incident, the mass of the Airbus A380 aircraft was 5,22,990 kg. Wind magnitude around the time of the event simulated using WRF is shown in figure 3.

The probable area of the persistence of the vortices descending from the Airbus A380 estimated using the

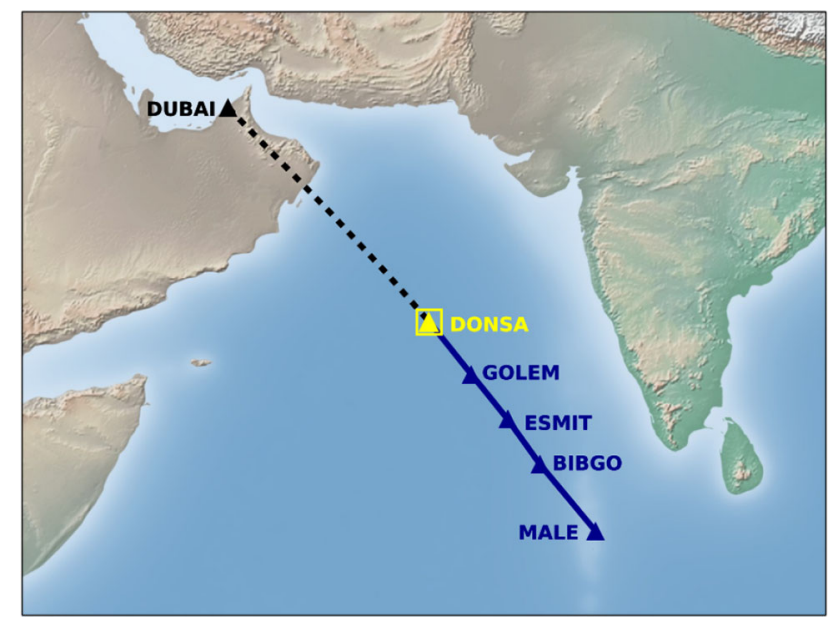

Figure 2. Flight path followed by the Canadair CL604 Challenger private jet and Airbus A380 aircraft. The black dotted line represents flight path of Airbus A380 starting from Dubai, and the blue line depicts the flight track of CL604 departing from Male. Triangles symbolize the waypoint followed by both the airplanes over the Arabian Sea. The rectangular box shows the area of the incident where the two aircraft crossed each other with 1000 feet vertical separation (adapted from the interim report [21]).

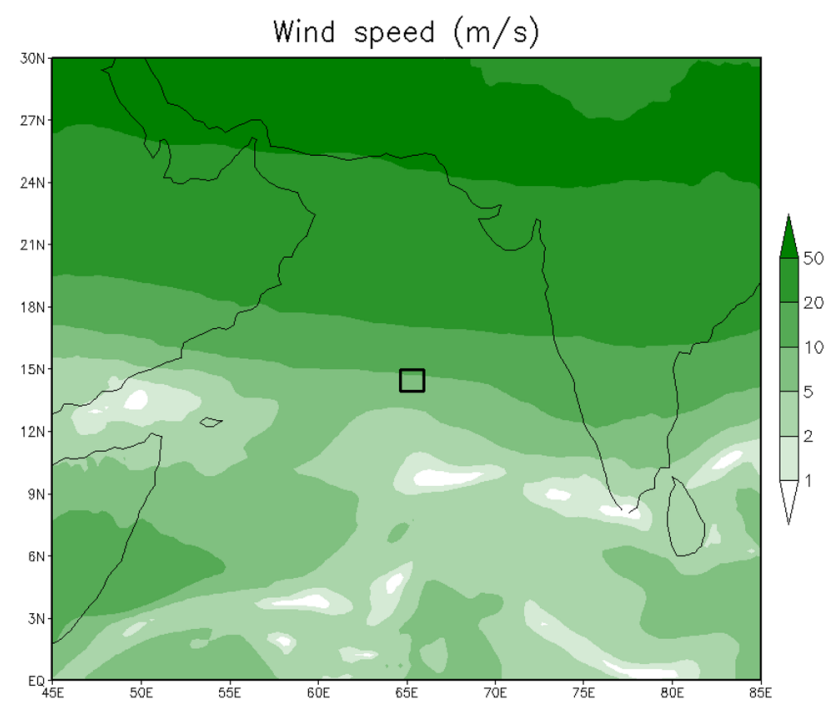

Figure 3. Large-scale wind field from WRF simulation at 08:30Z of $7^{\text {th }}$ January 2017 at an altitude of $10,500 \mathrm{~m}$. Rectangular box indicates the region of the incident.

potential vortex-hazard index at different $t$ along the flight path of Airbus A380 is shown in figure 4. At $t_{1}$ the Airbus A380 was flying near the region where $U \sim 22 \mathrm{~m} / \mathrm{s}$ and $q \sim 0.77 \mathrm{~m} / \mathrm{s}$ at 35,000 feet and $U \sim 23 \mathrm{~m} / \mathrm{s}$ and $q \sim 0.79 \mathrm{~m} / \mathrm{s}$ at 34,500 feet. At $t_{3}$ it was close to the area where $U \sim 2.7 \mathrm{~m} / \mathrm{s}$ and $q \sim 0.1 \mathrm{~m} / \mathrm{s}$ at 35,000 feet. One can see that the vortices descend and decay at different rates along the flight path of Airbus A380 due to the difference in the local atmospheric 


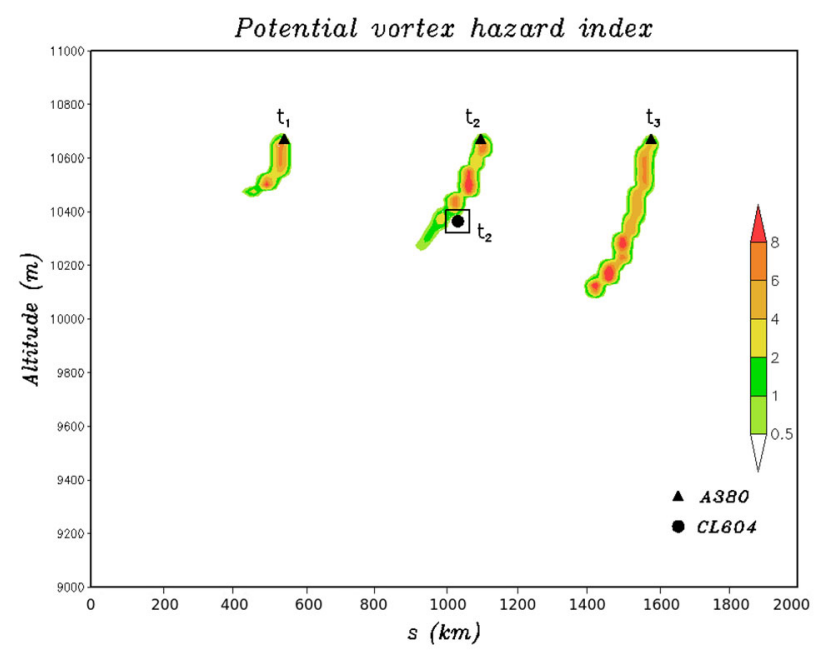

Figure 4. Potential vortex-hazard index along the distance $s$ from the flight path of Airbus A380. The track of Airbus A380 is from left to right, and CL604 is from right to left. Triangles represent the position of Airbus A380 and the circle shows the location of CL604 at different $t$ along their flight path. The region of the incident is marked using the rectangular box; $t_{1} \sim 08: 10 \mathrm{Z}$, $t_{2} \sim 08: 40 \mathrm{Z}$ and $t_{3} \sim 09: 10 \mathrm{Z}$.

conditions. The index is formulated in a way such that for a given $t$ it includes the cumulative effect of all vortices present in the particular flight grid. The increase in the intensity of the index (red shades in figure 4) is because descent velocity gets reduced over time and the vortices stay in that particular flight grid for a longer time. The atmospheric conditions of the event day are such that $q$ keeps on increasing with lower altitude, due to which the descent velocity gets reduced over time. Also, it is evident that the vortices descend more than 1000 feet or $300 \mathrm{~m}$ (minimum vertical separation) in a few places but not everywhere.

Around the time of the incident $t_{2}$, the local weather conditions at 35,000 feet where the Airbus A380 was flying were $U \sim 8.1 \mathrm{~m} / \mathrm{s}$ and $q \sim 0.36 \mathrm{~m} / \mathrm{s}$, due to which the vortices from Airbus A380 descended more than 1000 feet or $300 \mathrm{~m}$ and entered the incident zone (marked using the rectangular box in figure 4), where the private jet CL604 got affected and lost its control. Thus the potential vortexhazard method is successful in predicting the wake turbulence encounter at cruise level.

\subsection{A counter-example: $2^{\text {nd }}$ January 2018 crossing but no-encounter case}

A counter-example of the previous case where the superheavy category aircraft crossed a heavy category aircraft with 1000 feet vertical separation was found. There is no wake turbulence encounter report available in the public domain that intimates that there was a crossing, but no encounter occurred. On $2^{\text {nd }}$ January 2018, at about 20:13Z,

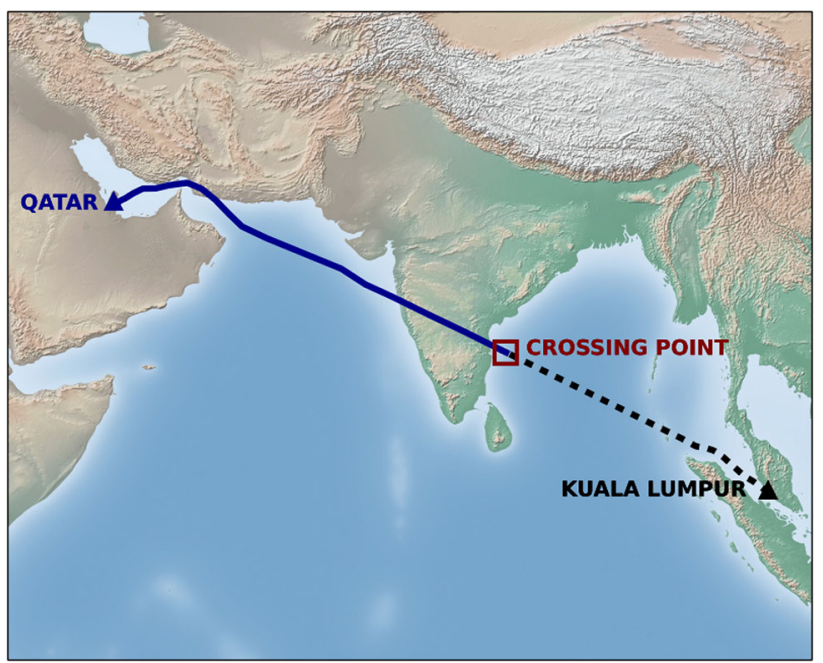

Figure 5. Flight path of Airbus A380 (black dotted line) departing from Kuala Lumpur and Airbus A330 (blue line) departing from Qatar. The rectangular box indicates the region where both the aircraft crossed each other with 1000 feet vertical separation and Airbus A380 is above Airbus A330.

Airbus A380 owned by the Emirates performing flight number UAE343 from Kuala Lumpur to Dubai [22] flying at 38,000 feet had crossed Airbus A330 belonging to Qatar Airways performing flight number QTR844 from Qatar to Kuala Lumpur [23] flying at 37,000 feet.

The flight path and crossing location of the two aircraft are shown in figure 5. Wind speed around the crossing time simulated using WRF is shown in figure 6. For this case, the mass of the Airbus A380 is assumed to be $5,00,000 \mathrm{~kg}$ since the maximum distance it travelled was less than $6000 \mathrm{~km}$ (less than the half of its operating range). Potential vortexhazard index calculated along the flight path of Airbus A380 at different $t$ is shown in figure 7. Around the time of crossing, the weather conditions at 38,000 feet where the Airbus A380 was flying are $U \sim 8.7 \mathrm{~m} / \mathrm{s}$ and $q \sim 0.63 \mathrm{~m} / \mathrm{s}$. Although the magnitude of wind was relatively close to the value of the previous case, the difference in the value of $q$ is because of the vertical wind shear, which is approximately two times the value of the previous wake turbulence encounter case. It is obvious that the vortices of Airbus A380 descending from 38,000 feet were not able to reach 37,000 feet, where the Airbus A330 was flying, because of the local atmospheric conditions. Hence the potential vortex-hazard index can provide a reasonable prediction of whether wake turbulence encounter will happen or not. Stratification, which is not considered in our model, could change some of the details, such as the vortex lifetime.

\section{$4.328^{\text {th }}$ November 2017 wake vortex encounter case}

On $28^{\text {th }}$ November 2017 [24], around 05:30Z, a Boeing 787-900 aircraft performing flight number UAL2 from 


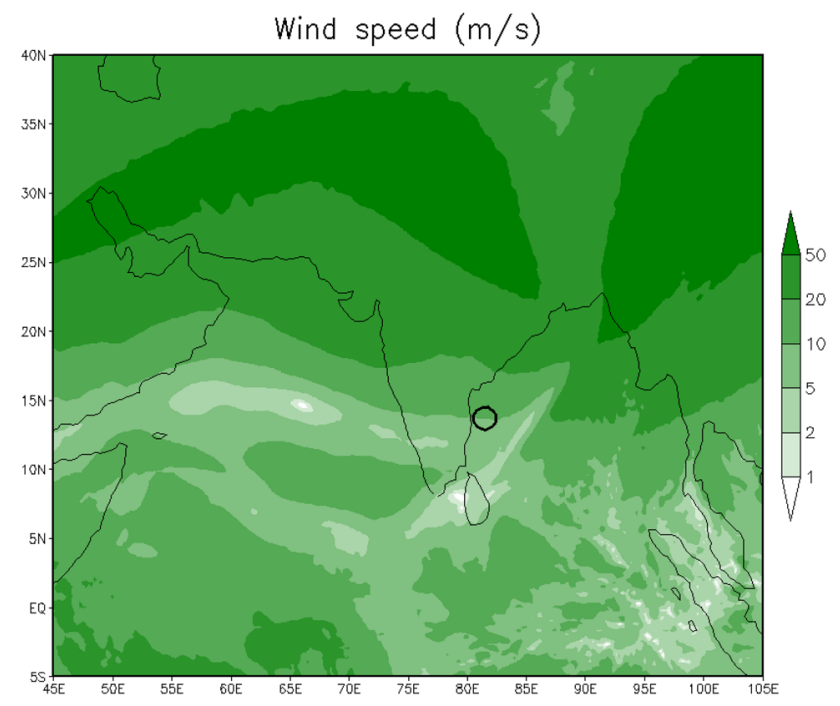

Figure 6. Large-scale wind field from WRF simulation at 20:00Z of $2^{\text {nd }}$ January 2018 at an altitude of $11,500 \mathrm{~m}$. The circle symbolizes the region where both the aircraft crossed each other without any wake turbulence encounter.

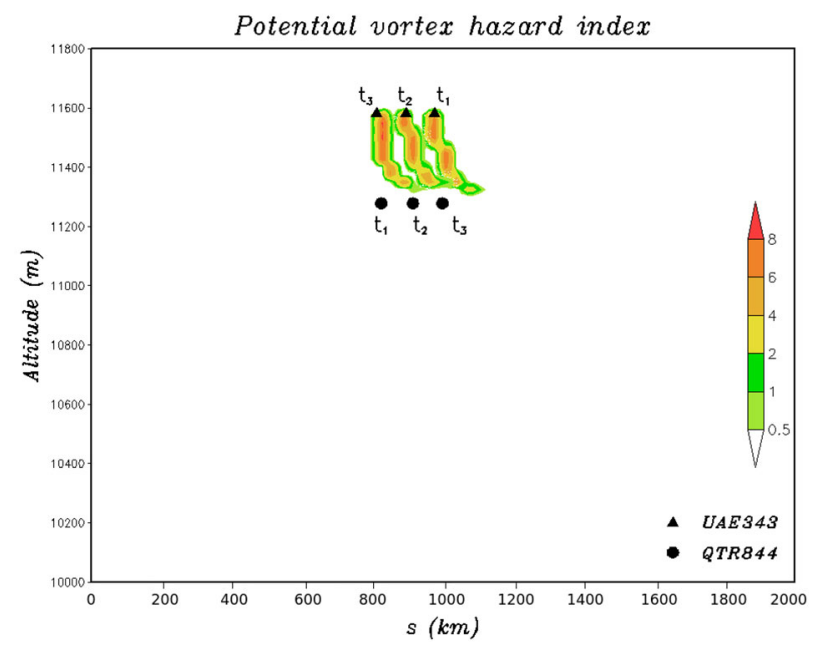

Figure 7. Potential vortex-hazard index along the distance $s$ from the flight path of Airbus A380 (UAE343). The track of Airbus A380 is from right to left, and Airbus A330 (QTR844) is from left to right. Triangles symbolize the position of Airbus A380 and circles point the location of Airbus A330 at different $t$ along their flight path; $t_{1} \sim 20: 07 \mathrm{Z}, t_{2} \sim 20: 13 \mathrm{Z}$ and $t_{3} \sim 20: 19 \mathrm{Z}$.

Singapore to San Francisco [25] had encountered wake turbulence of Boeing 747-400 performing flight number AAR702 from Manila to Seoul [26]. It happened near ABVAR waypoint at 36,000 feet. About 2 min before the event, Boeing 747-400 passed the crossing point at 37,000 feet. The Boeing 787-900 was diverted to Tokyo to take care of the injured passengers.

By tracking the flight path of Boeing 747-400 (AAR702), we found that another aircraft Boeing 777

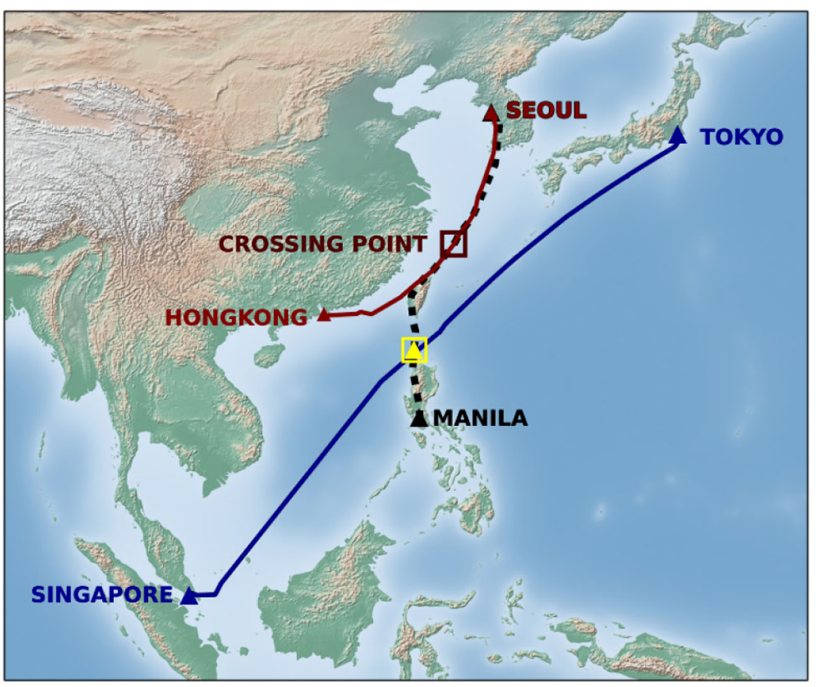

Figure 8. Flight path of Boeing 747-400 (black dotted line) departing from Manila, Boeing 787-900 (blue line) departing from Singapore and Boeing 777 (brown line) departing from Seoul. The region where the wake vortex encounter occurred is marked with the yellow rectangle. The brown rectangular box indicates the area where Boeing 747-400 crossed Boeing 777 aircraft with 1000 feet vertical separation.

performing flight number FDX5986 from Seoul to Hong Kong [27] had crossed the AAR702 around 06:34Z at 36,000 feet. For this crossing, there is no encounter report available in the public domain, even though the vertical separation between the two aircraft was 1000 feet, same as that of the Boeing 787-900 (UAL2) encounter case. It indicates that there was no wake turbulence encounter for Boeing 777 (FDX5986). The flight path of all three aircraft is shown in figure 8. Wind speed around the time of wake turbulence encounter simulated using WRF is shown in figure 9. At the incident time $t_{1}$, the local weather conditions at 37,000 feet where the Boeing 747-400 was flying are $U \sim 1.9 \mathrm{~m} / \mathrm{s}$ and $q \sim 0.15 \mathrm{~m} / \mathrm{s}$. The atmospheric conditions at 37,000 feet around $t_{4}$ where the Boeing $747-400$ was flying above the Boeing 777 without any wake turbulence encounter are $U \sim 36 \mathrm{~m} / \mathrm{s}$ and $q \sim 1.24 \mathrm{~m} / \mathrm{s}$.

The possible region of the persistence of vortices from Boeing 747-400 along its flight path at different $t$ is shown in figure 10. It is clear that at about 05:30Z, Boeing 787-900 encountered the descending vortices of Boeing 747-400 even though the vertical separation between the two aircraft was 1000 feet or $300 \mathrm{~m}$. However, at other locations where Boeing 777 flew beneath Boeing 747-400 with minimum vertical separation distance, the local weather conditions are such that the vortices descending from Boeing 747-400 decayed before reaching 36,000 feet. Thus, the potential vortex-hazard method is successful in predicting two different circumstances that happened along the corresponding flight path of Boeing 747-400 aircraft. 


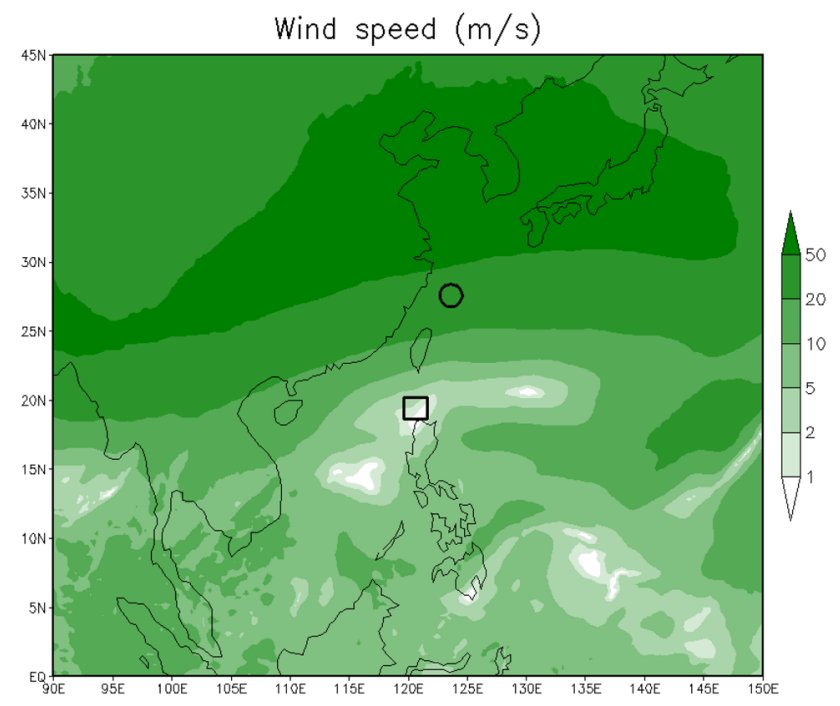

Figure 9. Wind speed from WRF simulation at $05: 30 \mathrm{Z}$ of $28^{\text {th }}$ November 2017 at an altitude of 11,250 m. Rectangular box indicates the region of wake turbulence encounter between Boeing 747-400 and Boeing 787-900. The circle shows the area where Boeing 747-400 and Boeing 777 aircraft crossed each other without any wake turbulence encounter.

\section{$4.42^{\text {nd }}$ January 2018 crossing but no encounter case}

On 2nd January 2018, at about 01:19Z, Airbus A380 owned by the Emirates Airlines performing flight number UAE419 from Bangkok to Dubai [28] flying at 38,000 feet had crossed Airbus A330 belonging to EgyptAir Airlines performing flight number MSR960 from Cairo to Bangkok [29] flying at 37,000 feet. Though the vertical separation between the two aircraft at the time of crossing was 1000 feet, there is no wake vortex encounter report available in the public domain. The flight path and crossing location of both the aircraft are shown in figure 11. Mass of Airbus A380 is assumed to be 5,75,000 kg (maximum takeoff weight). Wind magnitude around the crossing time simulated using WRF is shown in figure 12. Around the time of crossing, the local atmospheric conditions at 38,000 feet where the Airbus A380 was flying above the Airbus A330 are $U \sim 30 \mathrm{~m} / \mathrm{s}$ and $q \sim 1.0 \mathrm{~m} / \mathrm{s}$.

Potential vortex-hazard index evaluated along the flight path of Airbus A380 at different $t$ is presented in figure 13. Because of the prevailing atmospheric conditions, descending vortices of Airbus A380 from 38,000 feet were unable to reach 37,000 feet, where Airbus A330 was flying. Hence the index has potential to predict the no-encounter scenario.

\section{$4.54^{\text {th }}$ October 2014 wake vortex encounter case}

On 4th October 2014, a Jet Airways aircraft Boeing 737-800W encountered severe turbulence while performing

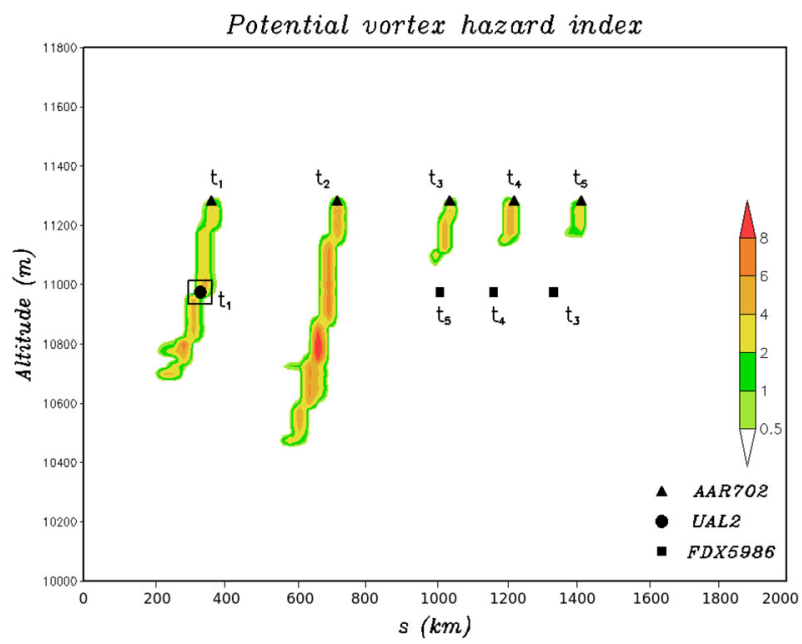

Figure 10. Potential vortex-hazard index along the distance $s$ from the flight path of Boeing 747-400. Triangles symbolize the position of Boeing 747-400 (AAR702), the circle shows the location of Boeing 787-900 (UAL2) and rectangles indicate the position of Boeing 777 (FDX5986) at different $t$ along their flight path. The track of Boeing 747-400 is from left to right. The region of the incident is marked using the rectangular box; $t_{1} \sim 05: 30 \mathrm{Z}$, $t_{2} \sim 05: 55 \mathrm{Z}, t_{3} \sim 06: 20 \mathrm{Z}, t_{4} \sim 06: 34 \mathrm{Z}$ and $t_{5} \sim 06: 48 \mathrm{Z}$.

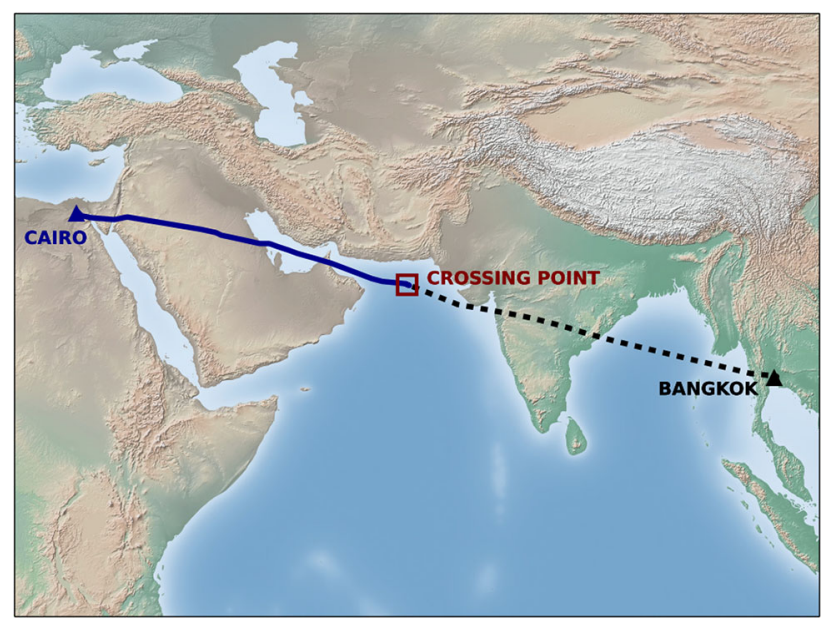

Figure 11. Flight path of Airbus A380 (black dotted line) departing from Bangkok and Airbus A330 (blue line) departing from Cairo. The rectangular box indicates the region where both the aircraft crossed each other with 1000 feet vertical separation and Airbus A380 is above Airbus A330.

flight number 9W565 from Dammam to Thiruvananthapuram. The information in the public domain [30] suggests CAT as the possible cause of the incident. Later, through private communication with the pilot of Boeing 737-800W aircraft [31], we found that the turbulence was due to the trailing vortices of Airbus A380 that passed above them in the opposite direction with 1000 feet vertical separation. The incident occurred at 23:11 $\mathrm{Z}$ when Boeing 737-800W was cruising at 37,000 feet over the Arabian Sea. The exact 


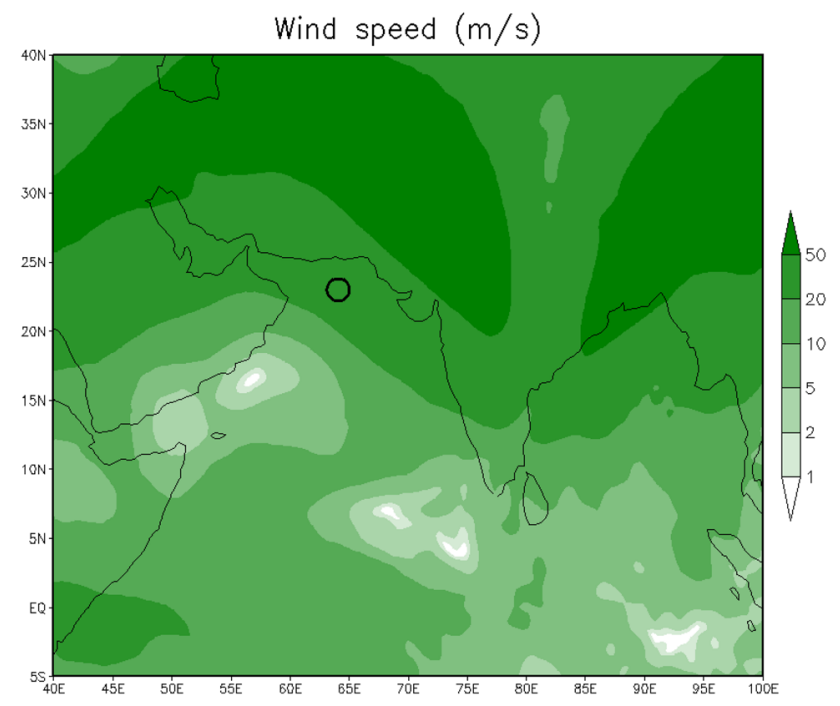

Figure 12. Wind magnitude from WRF simulation at 01:00Z of $2^{\text {nd }}$ January 2018 at an altitude of $11,500 \mathrm{~m}$. The region where both the aircraft crossed each other without any wake turbulence encounter is marked using the circle.

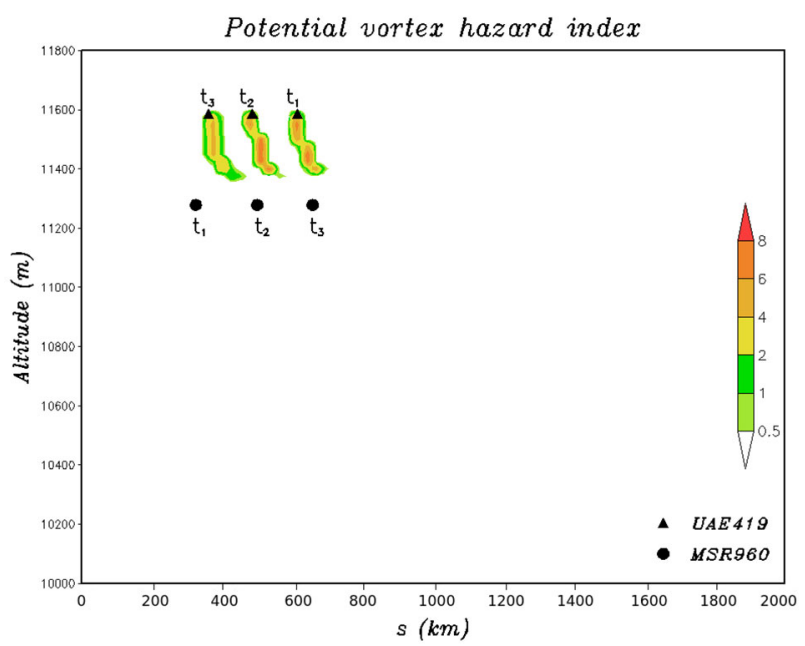

Figure 13. Potential vortex-hazard index along the distance from the flight path $s$ of Airbus A380 (UAE419). The track of Airbus A380 is from right to left, and Airbus A330 (MSR960) is from left to right. Triangles symbolize the position of Airbus A380 and circles point the location of Airbus A330 at different $t$ along their flight path; $t_{1} \sim 01: 09 \mathrm{Z}, t_{2} \sim 01: 19 \mathrm{Z}$ and $t_{3} \sim 01: 29 \mathrm{Z}$.

event position was $17.56 \mathrm{~N}$ and $63.99 \mathrm{E}$. The flight path followed by the Boeing $737-800 \mathrm{~W}$ aircraft is shown in figure 14.

Details of the Airbus A380 that caused wake vortex encounter are not known. The only handy information in the pilot report is that it was flying at 38,000 feet in the opposite direction of Boeing 737-800W. Since the departure and arrival locations of Airbus A380 are not known, we assume that Airbus A380 followed the flight path of Boeing $737-800 \mathrm{~W}$ in the opposite direction at 38,000 feet. Also, the

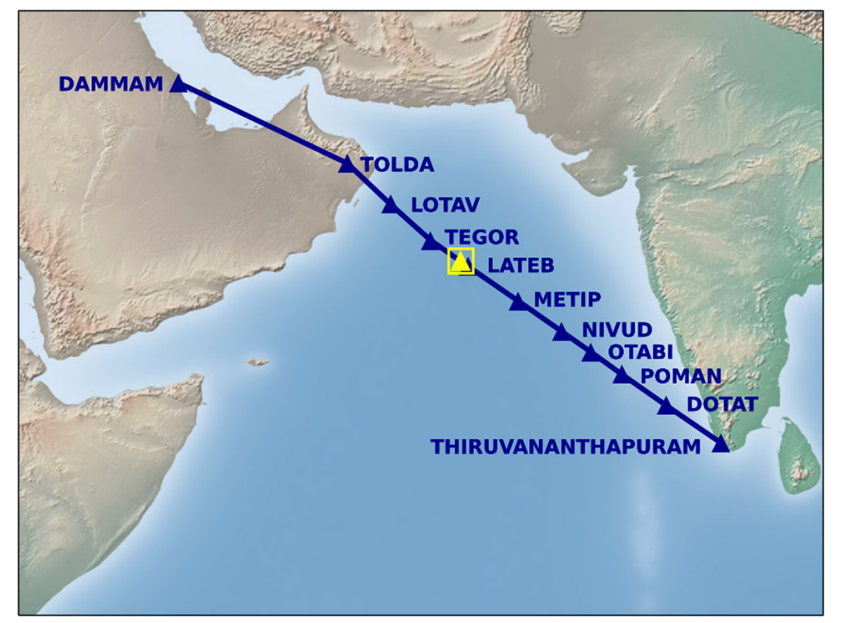

Figure 14. Flight path of Boeing 737-800W. The rectangular box indicates the location where Boeing $737-800 \mathrm{~W}$ encountered the trailing vortices of Airbus A380 (adapted from the pilot report [31]).

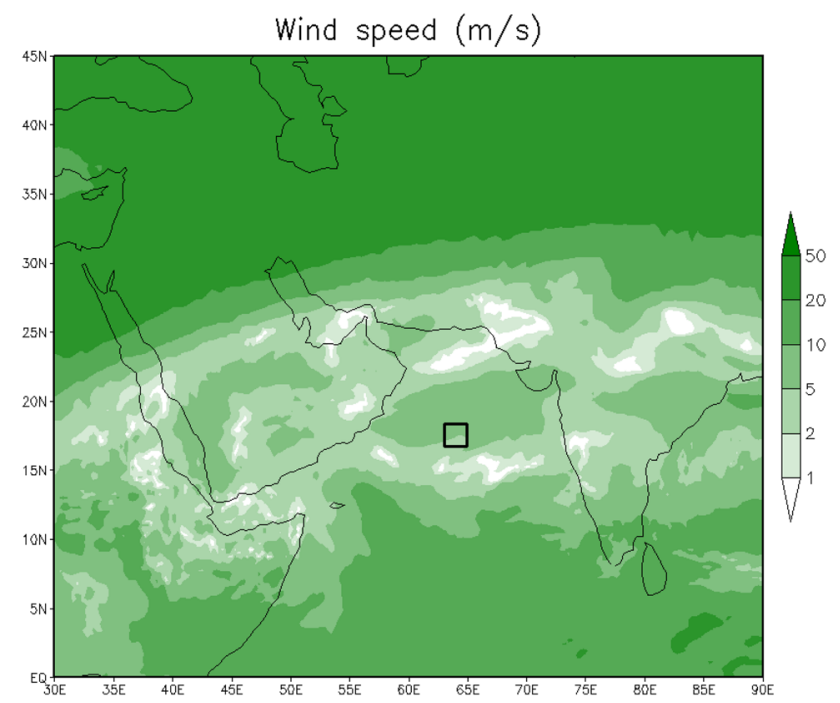

Figure 15. Wind magnitude from WRF simulation at 23:00Z of $4^{\text {th }}$ October 2014 at an altitude of $11,500 \mathrm{~m}$. Rectangular box indicates the region of the incident.

mass of Airbus A380 is considered to be $3,94,000 \mathrm{~kg}$ (maximum landing weight). Wind magnitude around the time of the event simulated using WRF is shown in figure 15. The local atmospheric conditions at 38,000 feet around the time of incident are $U \sim 4.3 \mathrm{~m} / \mathrm{s}$ and $q \sim 0.14 \mathrm{~m} / \mathrm{s}$.

The possible area of the persistence of vortices descending from 38,000 feet along the assumed flight path of Airbus A380 is shown in figure 16. It is evident that the vortices of Airbus A380 descended more than 1000 feet and caused the wake turbulence encounter.

Therefore the potential vortex-hazard method is successful in predicting all the scenarios considered in this study. Table 2 gives a summary of all the test cases chosen for this study. 


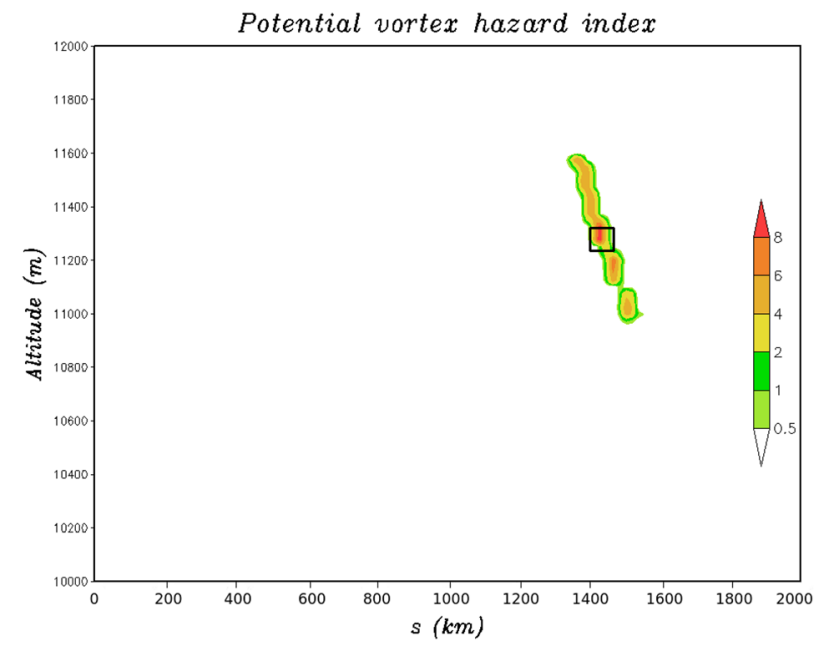

Figure 16. Potential vortex-hazard index along the distance $s$ from the assumed flight path of Airbus A380. The incident region is marked with the rectangular box.

Table 2. Summary of the test cases.

\begin{tabular}{|c|c|c|}
\hline Test cases & Details & Prediction \\
\hline \multicolumn{3}{|l|}{ Training cases } \\
\hline Case 1: $7^{\text {th }}$ Jan 2017 & Encounter & $\checkmark$ \\
\hline Case 2: $2^{\text {nd }}$ Jan 2018 & $\begin{array}{l}\text { Crossing but } \\
\text { no encounter }\end{array}$ & $\boldsymbol{\sim}$ \\
\hline \multicolumn{3}{|l|}{ Test cases } \\
\hline Case 1: $28^{\text {th }}$ Nov 2017 & Encounter & $\checkmark$ \\
\hline Case 2: $28^{\text {th }}$ Nov 2017 & $\begin{array}{l}\text { Crossing but } \\
\text { no encounter }\end{array}$ & $\checkmark$ \\
\hline Case 3: $2^{\text {nd }}$ Jan 2018 & $\begin{array}{l}\text { Crossing but } \\
\text { no encounter }\end{array}$ & $\checkmark$ \\
\hline Case 4: $4^{\text {th }}$ Oct 2014 & Encounter & $\checkmark$ \\
\hline
\end{tabular}

\section{Conclusion}

The issue of wake turbulence encounters has been studied extensively for take-off/landing conditions. Various methods have been proposed to determine the probability of a possible vortex encounter over the runway. The information available in the public domain shows that over the last few decades the problem of turbulence due to the trailing vortices of large aircraft has been increasing at cruise altitude. In this paper, we have developed a method to study whether wake turbulence encounters at cruise level will happen or not. The weather conditions for all the identified test cases were simulated using the WRF model.

As a first step, a model for assessing the wake turbulence encounter and cruise-level vortex encounters has been developed with various atmospheric effects on descending vortices. By combining the information obtained from the atmospheric model (WRF) and the proposed vortex model, a potential vortex-hazard index that shows the possible area of the persistence of the vortices at high altitude has been proposed. The usefulness of the index in predicting the wake turbulence encounter was examined with the test cases, and it was successful in establishing all different scenarios considered. The vortices from Airbus A380 and Boeing 747 aircraft descended more than 1000 feet or $300 \mathrm{~m}$ but not everywhere because of the prevailing atmospheric conditions. Inclusion of the effects of stratification would further enhance the model.

The proposed method has an advantage in that it includes the effect of all the vortices from different generating aircraft whenever the air-traffic density is high. An important feature of the index is that it can be used to forecast the turbulent regions due to the persistence of trailing vortices of large aircraft. With the forecast atmospheric conditions and details of Airbus A380 and Boeing 747 flying in the airspace, an estimate of the turbulent area can be obtained. The real-time prediction of such estimates could help the pilot to be aware of these turbulent regions, and flight safety with passenger comfort could be increased by avoiding them.

\section{Acknowledgements}

This work was carried out as a part of SERB/DST Project Number SB/S4/AS-145/2014. The support is gratefully acknowledged. The authors are thankful to Ms Asha V and Ms K Veena for their support. They also thank NCAR for making WRF available in the public domain and NCEP for providing the FNL datasets. They further thank Prof Joseph Mathew and Dr V Ramesh for their valuable comments. They appreciate the valuable comments and suggestions by the anonymous reviewer, which have led to improvements of this paper.

$\begin{array}{ll}\begin{array}{l}\text { Nomenclature } \\ b\end{array} & \text { wingspan of the generating aircraft }(\mathrm{m}) \\ e & \text { turbulence kinetic energy }(\mathrm{J} / \mathrm{kg}) \\ g & \text { acceleration due to gravity }\left(\mathrm{m} / \mathrm{s}^{2}\right) \\ I & \text { atmospheric turbulence intensity percentage } \\ k_{s} & \text { constant for shear production } \\ l_{s} & \Delta z, \text { length scale for shear production }(\mathrm{m}) \\ m & \text { mass of the generating aircraft }(\mathrm{kg}) \\ q & \text { measure of the turbulence kinetic energy }(\mathrm{m} / \mathrm{s}) \\ R_{12} & \text { initial separation distance between the vortices } \\ & \text { (m); for elliptical loading, }(\pi / 4) b \\ r_{12} & \begin{array}{l}\text { half of the initial separation distance between } \\ \text { the vortices (m) }\end{array} \\ s & \begin{array}{l}\text { distance along the flight path of the generating } \\ \text { aircraft (km) }\end{array} \\ t & \begin{array}{l}\text { time in UTC } \\ \text { magnitude of atmospheric velocities along the }\end{array} \\ U & \begin{array}{l}\text { flight path of the generating aircraft (m/s) } \\ \text { velocity component of wind in } x, y \text { and }\end{array} \\ & z \text { directions, } \\ u_{p}, v_{p}, w_{p} & \text { respectively, along the flight path of the } \\ \text { generating aircraft (m/s) }\end{array}$




\begin{tabular}{|c|c|}
\hline$V_{c}$ & cruise velocity of the generating aircraft $(\mathrm{m} / \mathrm{s})$ \\
\hline$V_{E}$ & $\begin{array}{l}\text { cross-wind along the flight path of the } \\
\text { generating aircraft }(\mathrm{m} / \mathrm{s})\end{array}$ \\
\hline$V_{T}$ & $\begin{array}{l}\text { tail wind along the flight path of the } \\
\text { generating aircraft }(\mathrm{m} / \mathrm{s})\end{array}$ \\
\hline$w$ & $\begin{array}{l}\text { descent velocity of the vortices at any } \\
\text { given } \tau(\mathrm{m} / \mathrm{s})\end{array}$ \\
\hline$w_{0}$ & initial descent velocity $(\mathrm{m} / \mathrm{s})$ \\
\hline$x_{c}, y_{c}, z_{c}$ & centre of the elliptical area enclosing air masses \\
\hline$y_{1}, y_{2}$ & $\begin{array}{l}\text { lateral position of the port and starboard } \\
\text { vortices, respectively }\end{array}$ \\
\hline$z_{1}, z_{2}$ & $\begin{array}{l}\text { vertical position of the port and starboard } \\
\text { vortices, respectively }\end{array}$ \\
\hline$\Gamma$ & $\begin{array}{l}\text { circulation value of the vortices at any } \\
\text { given } \tau\left(\mathrm{m}^{2} / \mathrm{s}\right)\end{array}$ \\
\hline$\Gamma_{0}$ & initial circulation value of the vortices \\
\hline$\Gamma_{1}, \Gamma_{2}$ & $\begin{array}{l}\text { circulation value of the port and starboard } \\
\text { vortices, respectively }\end{array}$ \\
\hline$\Theta$ & angle of rotation \\
\hline$\theta$ & aircraft heading concerning the positive $x$-axis \\
\hline$\rho_{c}$ & density at cruise altitude $\left(\mathrm{kg} / \mathrm{m}^{3}\right)$ \\
\hline$\tau$ & time in seconds (s) \\
\hline$\tau_{\max }$ & $\begin{array}{l}\text { maximum time the vortices can } \\
\text { persist in the atmosphere (s) }\end{array}$ \\
\hline$\varphi, \lambda, h$ & $\begin{array}{l}\text { latitude, longitude and altitude of the } \\
\text { generating aircraft, respectively, at any given } t\end{array}$ \\
\hline & potential vortex-hazard index \\
\hline & angular velocity \\
\hline
\end{tabular}

\section{References}

[1] Evans J K 2014 An updated examination of aviation accidents associated with turbulence, wind shear and thunderstorm. AMA Report Number 14-14

[2] Nelson R C 2006 Trailing vortex wake encounters at altitude-a potential flight safety issue. AIAA Paper 2006-6268

[3] Nelson R C 2004 The trailing vortex wake hazard: beyond the takeoff and landing corridors. AIAA 2004-5171

[4] Bauer T, Vechtel D, Abdelmoula F and Immisch T 2014 Inflight wake encounter prediction with the wake encounter avoidance and advisory system. AIAA Paper 2014-2333

[5] Solch I, Holzapfel F, Abdelmoula F and Vechtel D 2016 Performance of on-board wake vortex prediction systems employing various meteorological data sources. J. Aircr. 53(5): 1505-1516

[6] Federal Aviation Administration 2014 Safety alert for operators (SAFO). SAFO 14007

[7] Federal Aviation Administration 2014 Advisory circular. AC No: $90-23 \mathrm{G}$

[8] Miloud K B, Dghim M, Fellouah H and Ferchichi M 2017 Experimental investigation on the effect of free stream turbulence on wingtip vortices. AIAA 2017-3037

[9] Donaldson C duP and Bilanin A J 1975 Vortex wakes of conventional aircraft. AGARD-AG-204
[10] Hill F M 1975 A numerical study of the descent of a vortex pair in a stably stratified atmosphere. J. Fluid Mech. 71(1): $1-13$

[11] Holzapfel F 2014 Effects of environmental and aircraft parameters on wake vortex behavior. J. Aircr. 51(5): 1490-1500

[12] Delisi D P and Robins R E 2006 Effects of crosswind shear on trailing vortex evolution. AIAA 2006-1075

[13] Robins R E and Delisi D D 2006 Modeling crosswind shear effects in NASA's AVOSS prediction algorithm. In: Proceedings of the 44th AIAA Aerospace Sciences Meeting and Exhibit, AIAA 2006-1076

[14] Proctor F H and Ahmad N N 2011 Crosswind shear gradient affect on wake vortices. AIAA 2011-3038

[15] Hallock J N and Holzapfel F 2018 A review of recent wake vortex research for increasing airport capacity. Prog. Aerosp. Sci. 98: 27-36

[16] Venkatesh T N, Arshad Shameem C and Saravanakumar J 2017 Study of a possible wake vortex encounter of an aircraft over the Arabian Sea. J. Phys. Conf. Ser. 822(1): 012049

[17] Saravanakumar J, Arshad Shameem C and Venkatesh T N 2016 Development of an aircraft wake vortex model for assessing cruise-level encounters. NAL PD-CTFD/2016/ 1010

[18] Saravanakumar J, Arshad Shameem C and Venkatesh T N 2017 Calculation of a potential vortex hazard index at cruiselevel. In: Proceedings of the 19th AeSI Annual CFD Symposium, pp. 168-171

[19] Saravanakumar J, Arshad Shameem C and Venkatesh T N 2017 Case studies of cruise-level aircraft wake vortex encounters using the potential vortex-hazard index. NAL PD-CTFD/2017/1017

[20] Michalakes J, Dudhia J, Gill D, Henderson T, Klemp J B, Skamarock W C and Wang W 2005 The weather research and forecast model: software architecture and performance. In: Proceedings of the Eleventh ECMWF Workshop on the Use of High Performance Computing in Meteorology

[21] BFU 2017 German Federal Bureau of Aircraft accident investigation 2017 interim report. BFU17-0024-2X

[22] https://flightaware.com/live/flight/UAE343/history/ 20180102/1710Z/WMKK/OMDB

[23] https://flightaware.com/live/flight/QTR844/history/20180102/ 1610Z/OTHH/WMKK

[24] http://avherald.com/h?article=4b1a70a9

[25] https://flightaware.com/live/flight/UAL2/history/20171128/ 0200Z/WSSS/KSFO

[26] https://flightaware.com/live/flight/AAR702/history/20171128/ 0430Z/RPLL/RKSI

[27] https://flightaware.com/live/flight/FDX5986/history/20171128/ $0501 \mathrm{Z}$

[28] https://flightaware.com/live/flight/UAE419/history/20180101/ 2000Z/VTBS/OMDB

[29] https://flightaware.com/live/flight/MSR960/history/20180101/ 2120Z/HECA/VTBS

[30] http://timesofindia.indiatimes.com/city/mumbai/Aircraft-enco untered-turbulence-over-Bangalore/articleshow/5857479.cms

[31] Pilot report about incident on VT-JBU. Private communication 